\title{
Transient Hybridization Between Different Hemoglobins Detected by Cross Electrophoresis
}

\author{
Lihong Han ${ }^{1}$, Bin Yan ${ }^{1}$, Yan Su ${ }^{2}$, Liangyi Qin ${ }^{3}$, Lijun Gao ${ }^{2}$, Lishe Zhou ${ }^{2}$, Wenbin Qin ${ }^{2 *}$ \\ ${ }^{1}$ Department of Basic Medical Science,Putian University,Putian Fujian 351100, China \\ ${ }^{2}$ Laboratory of Hemoglobin, Baotou Medical College, Baotou, China \\ ${ }^{3}$ Clinical laboratory of Nanhui central hospital, Shanghai, China \\ Email: vhanlihong@163.com
}

\begin{abstract}
In this paper, we aimed to introduce a new method in detecting transient hemoglobin $(\mathrm{Hb})$ hybrids. First, electrophoretic pure $\mathrm{HbA}, \mathrm{HbA}_{2}, \mathrm{HbF}$ and $\mathrm{HbA}_{1}$ were prepared by starch-agar mixed gel, and then cross electrophoresis was performed on $2 \%$ starch-agar mixed gel. The slow-moving $\mathrm{HbA}_{2}$ samples were added in the front long slots and the fast-moving $\mathrm{HbA}, \mathrm{HbF}$ and $\mathrm{HbA}_{1}$ samples were added in the back short slots of the gel. These two rows of slots are all located on the cathode side of the gel and about $1 \mathrm{~cm}$ away from each other. After electrophoresis, which was performed at $5 \mathrm{~V} / \mathrm{cm}$ for about $4 \mathrm{~h}$, the gel was stained with Ponceau Red and Benzidine. The results showed that when fast-moving $\mathrm{HbA}$ and $\mathrm{HbF}$ crossed slow-moving $\mathrm{HbA}_{2}$, the band shape of $\mathrm{HbA}_{2}$ was deformed as a valley, however when fast-moving $\mathrm{HbA}_{1}$ crossed slow-moving $\mathrm{HbA}_{2}$, the band shape of $\mathrm{HbA}_{2}$ was not deformed. This finding demonstrates that $\mathrm{HbA}$ and $\mathrm{HbF}$ could form transient hybrid with $\mathrm{HbA}_{2}$ during cross electrophoresis; however $\mathrm{HbA}_{1}$ could not form any hybrid with $\mathrm{HbA}_{2}$ because of its chemical modification of $\mathrm{Hb}$ subunits.

Keywords: cross electrophoresis, hemoglobin, transient hybrid
\end{abstract}

\section{Introduction}

Hemoglobin $(\mathrm{Hb})$ is a well-researched oxygen carrying protein in red blood cells (RBCs). There are $\mathrm{HbA}_{1} \mathrm{HbA}, \mathrm{HbF}$ and $\mathrm{HbA}_{2}$ in adult RBCs. $\mathrm{HbA}_{1}$ is relatively complicated, it is located in the anode side of $\mathrm{HbA}$ during electrophoresis and includes some modified products of $\mathrm{Hb}$, such as glycosylated $\mathrm{Hb}\left(\mathrm{HbA}_{\mathrm{lc}}\right)$ and glutathione combined $\mathrm{Hb}$. Glycosylated $\mathrm{Hb}$ is formed in a non-enzymatic pathway by Hb's normal exposure to high plasma levels of glucose and glutathione combined $\mathrm{Hb}$ is formed during storage ${ }^{[1]}$. All $\mathrm{Hbs}$ are made up of two kinds of similar globular proteins which polymerize together to form a tetramer. The subunit compositions of $\mathrm{HbA}, \mathrm{HbF}$ and $\mathrm{HbA}_{2}$ are $\alpha_{2} \beta_{2}, \alpha_{2} \gamma_{2}$ and $\alpha_{2} \delta_{2}$, respectively. Four subunits are bound to each other by salt bridges, hydrogen bonds, and hydrophobic interactions. There are two kinds of contacts between $\alpha$ and $\beta$ subunits: $\alpha_{1} \beta_{1}$ and $\alpha_{1} \beta_{2}$. Hybridization of Hbs is a more widespread phenomenon and is found in artificial mixtures of different Hbs. This kind of hybridization is generally called asymmetric hybridization. This hybridization happens when two kinds of $\mathrm{Hbs}$ are put together, after dissociation and recombination, different Hbs would exchange subunit and generate new hybrid molecule ${ }^{[2-4]}$. Thus, asymmetric hybridization has nothing to do with electrophoresis; however, electrophoresis technique can be used to identify the hybrid molecules. In our previous study, we had found for the first time that $\mathrm{HbA}$ could interact with $\mathrm{HbA}_{2}$ during cross electrophoresis ${ }^{[5]}$. We called this interaction as transient hybridization. In this study, we will continue to study the transient hybridization between $\mathrm{HbA}_{2}$ and other $\mathrm{Hbs}$, and try to understand its molecular mechanism and further application.

\section{Materials and methods}

\subsection{Blood source}

This study had been approved by our local ethics committee. Healthy adult blood, umbilical cord blood and diabetes blood were collected from the first affiliated hospital of Baotou medical college. Before the blood samples were collected, all the people who took part in this experiment were asked to sign the consent information. Venous blood samples were anti-coagulated with heparin, stored at $4{ }^{\circ} \mathrm{C}$, and generally analyzed within $24 \mathrm{~h}$.

\subsection{Preparation of hemolytic fluid of red blood cell}

Anti-coagulated venous blood from different individuals was centrifuged at $3000 \mathrm{rpm}$ for $10 \mathrm{~min}$ and the upper plasma was aspirated. To $1 \mathrm{ml}$ saline, was added $200 \mu \mathrm{l}$ of the lower RBCs. The mixture was centrifuged at $3000 \mathrm{rpm}$ for $10 \mathrm{~min}$, after which the supernatant was aspirated. This washing operation was repeated 4-5 times, and then $200 \mu 1$ water 
and $100 \mu \mathrm{l} \mathrm{CCl} \mathrm{C}_{4}$ were added to the RBCs. After vigorous vortex, the sample was centrifuged at $12000 \mathrm{rpm}$ for $10 \mathrm{~min}$ and the upper red hemolysate was piped out carefully for storage at $4^{\circ} \mathrm{C}$.

\subsection{Preparation of pure $\mathrm{HbA}, \mathrm{HbA} 1, \mathrm{HbA} 2$ and $\mathrm{HbF}$}

Electrophoretic pure $\mathrm{HbA}, \mathrm{HbA}_{1}, \mathrm{HbA}_{2}$ and $\mathrm{HbF}$ were preparaed by $2 \%$ starch-agar mixed gel (starch:agar=4:1) electrophoresis as described earlier ${ }^{[6]}$. After electrophoresis, the red bands of $\mathrm{HbA}, \mathrm{HbA}_{2}, \mathrm{HbA}_{1}$ and $\mathrm{HbF}$ were cut down from the gel and stored in an Eppendroff tube at $-80^{\circ} \mathrm{C}$, respectively. Before use, the gels were thawed at room temperature and centrifuged at $10000 \mathrm{rpm}$ for $10 \mathrm{~min}$, and then the red supernatants were piped out for further use.

\subsection{Cross electrophoretic technique}

Cross electrophoresis was performed on a $2 \% 10 \times 20 \mathrm{~cm}$ starch-agarose mixed gel, which was prepared as earlier ${ }^{[6]}$. Then two rows of sample slots about $1 \mathrm{~cm}$ apart, were made on the cathodic side of the gel which was about $1.5 \mathrm{~cm}$ away from the edge as indicated in Figure 1. The front row is made up of long slots, and the back row is made up of short slots. The back short slots were just behind the front long slots and at each side of cross electrophoresis group, control slots were made. 10-15 $\mu \mathrm{L}$ electrophoretic pure $\mathrm{Hb}$ samples were subsequently applied on the slots using $3 \mathrm{MM}$ filter paper. Generally, the slow-moving Hbs were added in the front row and the fast-moving Hbs were added in the back row. Electrophoresis was performed in borate buffer at $5 \mathrm{~V} / \mathrm{cm}$ for $4 \mathrm{~h}$. After electrophoresis, we first observed the red bands on the gel directly, and then stained the gel as usual ${ }^{[6]}$.

\section{Results}

\subsection{Example diagram of cross electrophoretic}

In our study, starch-agar mixed gel electrophoresis was used to prepare electrophoretic pure $\mathrm{HbA}, \mathrm{HbA}_{2}, \mathrm{HbA}_{1}$ and $\mathrm{HbF}$. Then, cross electrophoresis was performed to observe the interaction between slow-moving $\mathrm{HbA}_{2}$ and fast-moving $\mathrm{HbA}, \mathrm{HbA}_{1}$ and $\mathrm{HbF}$, respectively. If there is interaction between each other, the central part of $\mathrm{HbA}_{2}$ band will bend toward the anode as a valley. Otherwise, the band shape will not be deformed. As shown in Figure 1, when HbA threaded $\mathrm{HbA}_{2}$, the long $\mathrm{HbA}_{2}$ band bent toward the anode as a valley. The same phenomenon appeared when $\mathrm{HbF}$ threaded $\mathrm{HbA}_{2}$, showing that $\mathrm{HbF}$ may be interacting with $\mathrm{HbA}_{2}$. However, when $\mathrm{HbA}_{1}$ threaded $\mathrm{HbA}_{2}$, the band shape of $\mathrm{HbA}_{2}$ did not change; indicating that $\mathrm{HbA}_{1}$ may have no interaction with $\mathrm{HbA}_{2}$.

\subsection{Coarse structures of $\mathbf{H b s}$}

Coarse structures of $\mathrm{HbA}, \mathrm{HbA}_{2}$ and $\mathrm{HbF}$ are $\alpha_{2} \beta_{2}, \alpha_{2} \delta_{2}$ and $\alpha_{2} \gamma_{2}$, respectively. There has been an 'asymmetric hybridization' with regard to the molecular hybridization of $\mathrm{Hb}^{[3,4]}$. Its reaction formula is assumed as follows in the case of $\mathrm{HbA}: \alpha_{2}^{\mathrm{A}} \beta^{\mathrm{A}}{ }_{2}+\alpha^{\mathrm{B}}{ }_{2} \beta_{2}^{\mathrm{B}} \rightarrow \alpha^{\mathrm{A}}{ }_{2}+\beta^{\mathrm{A}}{ }_{2}+\alpha^{\mathrm{B}}{ }_{2}+\beta^{\mathrm{B}}{ }_{2} \rightarrow \alpha_{2}^{\mathrm{A}} \beta^{\mathrm{B}}{ }_{2}+\alpha^{\mathrm{B}}{ }_{2} \beta^{\mathrm{A}}$. In this formula, $\alpha^{\mathrm{A}}{ }_{2} \beta_{2}^{\mathrm{A}}$ represents human normal $\mathrm{HbA}$ and $\alpha_{2}^{\mathrm{B}} \beta_{2}^{\mathrm{B}}{ }_{2}$ represents animal $\mathrm{HbA}$ variant. After putting two kinds of Hbs together, they will exchange subunit and generate new hybrid molecules. Asymmetric hybridization has nothing to do with electrophoresis; however, electrophoretic technique can be used to identify the hybrid molecules.

\section{Discussion}

In our study, the hybridization of Hbs is presumed as 'transient hybridization', and the specific reaction formula of $\mathrm{HbA}$ and $\mathrm{HbA}_{2}$ is assumed as follow: $\alpha_{2} \beta_{2}+\alpha_{2} \delta_{2} \longleftrightarrow(\alpha \beta)_{2}+(\alpha \delta)_{2} \longleftrightarrow 2(\alpha \beta: \alpha \delta)$. This progress occurred during electrophoresis when fast-moving Hbs cross slow-moving ones. Thus, transient hybridization belongs to 'dynamic hybridization' of hemoglobin. However, the binding force between the subunits of $\alpha \beta: \alpha \delta$ was so weak that they gradually dissociated during electrophoresis, causing the middle part of $\mathrm{HbA}_{2}$ band to move faster and form the special "valley" shape. In contrast with $\mathrm{HbA}$ and $\mathrm{HbF}$, the composition of $\mathrm{HbA}_{1}$ is more complex, which is a mixture of some modification products of $\mathrm{Hb}$, such as glycosylated $\mathrm{Hb}$ and glutathione combined $\mathrm{Hb}$, etc. This may have an effect on the interaction between $\mathrm{HbA}_{1}$ and $\mathrm{HbA}_{2}$, so we could not see the change of the $\mathrm{HbA}_{2}$ band after electrophoresis.

As to cross electrophoresis, in 1953, Grassmann and Hubner reported a method to detect the formation of loosely bound addition compound using the technique of continuous paper electrophoresis. They proposed the use of this method in the detection of biologically important reactions, such as the formation of antigen-antibody complexes and of enzyme-substrate complexes. Later, this method was named "cross electrophoresis" by Nakamura ${ }^{[7]}$. In our study, we initially performed cross electrophoresis on starch-agar gel to detect the interaction between different Hbs. We found that interaction may exist between some Hbs and be absent in other Hbs, the reason was speculated to have a relationship with the homologous and modification of Hbs. We believe that this method can be used to study not only the interaction 
between different human Hbs, but also the Hbs molecular evolution of different species.

\section{Acknowledgments}

This work was supported by the Fund of Higher Education Scientific Research in Inner Mongolia Autonomous Region (NJZY16200), Putian University's Introduction of Talent Research Startup Project (2018091) and Middle-aged and Young People Backbone Project of Fujian Health Commission (2019-ZQNB-18). We especially acknowledge all of the people who donate their blood samples for our research.

Conflict of interest statement: The authors declare no competing financial interests.

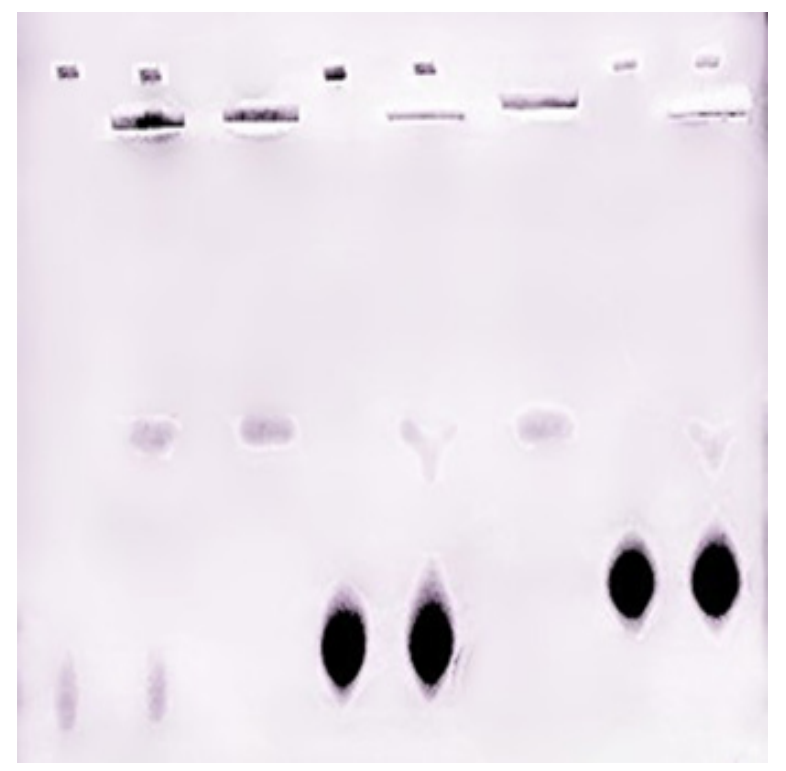

Figure 1. Transient hybrid between different $\mathrm{Hbs}$. Lane 1,3,4,6 and 7 are control samples of $\mathrm{HbA}_{1}, \mathrm{HbA}_{2}, \mathrm{HbA}, \mathrm{HbA}$ and $\mathrm{HbF}$, respectively. Lane 2 , 5 and 8 are hybrid groups between $\mathrm{HbA}_{1}$ and $\mathrm{HbA}_{2}, \mathrm{HbA}$ and $\mathrm{HbA}_{2}, \mathrm{HbF}$ and $\mathrm{HbA}_{2}$, respectively. Electrophoresis was performed in borate buffer at $5 \mathrm{~V} / \mathrm{cm}$ for $4 \mathrm{~h}$

\section{References}

[1] Jeppsson JO, Jerntorp P, Almër LO, Persson R, Ekberg G, Sundkvist G. Capillary blood on filter paper for determination of HbA1c by ion exchange chromatography. Diabetes Care. 1996; 19(2): 142-145.

[2] Itano HA, Robinson E. Properties and inheritance of haemoglobin by asymmetric recombination. Nature. 1959; 184: 1468-1469.

[3] Chao TC, Chin WP, Wu YH. Interspecific "hybridization" of several mammalian haemoglobins. Sci Sin. 1966; 15(3): 360-370.

[4] Efremov GD, Huisman TH, Smith LL, Wilson JB, Kitchens JL, Wrightstone RN, Adams HR. Hemoglobin Richmond, a human hemoglobin which forms asymmetric hybrids with other hemoglobins. Biol Chem. 1969; 244(22): 61056116.

[5] Qin WB. Extra-cellular Interaction between $\mathrm{HbA}_{2}$ and HbA. Chinese Biochemical Journal. 1991; 7: 583-584.

[6] Su Y, Shao G, Gao LJ, Zhou LS, Qin LY, Qin WB. RBC electrophoresis with discontinous power supply - a newly established hemoglobin release test. Electrophoresis. 2009; 30: 1-4.

[7] Nakamura S. Cross electrophoresis, its principle and applications. Korean Biochem. 1969; 2(1): 1-18. 